la estancia hospitalaria fue breve al posibilitar un alta precoz a las $24 \mathrm{~h}$ de la intervención igual que nuestro equipo realiza con las dilataciones de la estenosis pieloureterales congénitas ${ }^{6}$, y la morbilidad fue mínima al no dañar la vascularización del uréter. Entre sus incovenientes encontramos la dificultad para tutorizar la vía urinaria de un lactante, aunque pensamos que es posible disponiendo del material adecuado y tras una adecuada curva de aprendizaje. La necesidad de colocar un stent tipo doble J no representa una diferencia con la técnica abierta en la que también se utiliza rutinariamente ${ }^{4}$.

B I B L I O G R A F Í A

1. Smith BG, Metwalli AR, Leach J, Cheng EY, Kropp BP. Congenital midureteral stricture in chidren diagnosed with antenatal hydronephrosis. Urology. 2004;64:1014-9.

2. Campbell MF. Clinical considerations of the anatomy, physiology, embryology, and anomalies of the urogenital tract. In: Pediatric Urology. New York: MacMillan Co.; 1937. pp. 188, 273, 287-289.
3. Prieto JC, Castellan M, Gosalbez R, Labbie A, Perez-Brayfield M. Severe congenital midureteral dilatation. J Ped Surg. 2007;42: 257-8.

4. Hwang AH, McAleer IM, Shapiro E, Miller OF, Krous HF, Kaplan GW, et al. Congenital mid ureteral strictures. J Urol. 2005;174:1999-2002.

5. Gnessin E, Yossepowitch O, Holland R, Liune PM, Lifshitz DA. Holmium laser endoureterotomy for benign ureteral stricture: A single center experience. J Urol. 2009;182:2775-9.

6. Parente A, Angulo JM, Romero R, Rivas S, Laín A, Fanjul M, et al. Resultados a medio plazo del tratamiento endourológico retrógrado con balón de la estenosis pieloureteral en niños menores de 1 año. Actas Urol Esp. 2009;33:422-8.

A. Parente Hernández*, J.M. Angulo Madero,

R.M. Romero Ruiz y S. Rivas Vila

Sección Urología Pediátrica, Servicio Cirugía Pediátrica, Hospital Infantil Gregorio Marañón, Madrid, España

*Autor para correspondencia.

Correo electrónico: parente80@hotmail.com

(A. Parente Hernández).

\title{
Priapismo secundario a tratamiento por cabergolina: primera descripción de esta asociación
}

\section{Priapism secondary to treatment due to cabergoline: The first description of this association}

\section{Sr. Director:}

Recientemente hemos tratado en el servicio de urgencias a un paciente de 33 años por un cuadro de priapismo debajo flujo de seis horas de evolución. Entre su antecedentes solo destacaba que padecía de un hiperprolactinoma y estaba siendo tratado con cabergolina.

El paciente nos relató que el cuadro se había producido en 2 ocasiones antes del episodio que le obligó a asistir a urgencias. El primer episodio de erección prolongada se había producido al mes de iniciar el tratamiento habiéndose autolimitado de forma espontánea en 3 horas. El segundo episodio tuvo lugar una semana antes del definitivo, cursando de la misma forma que el previo.

En nuestra asistencia se realizaron las maniobras monitorizadas de inyección de epinefrina, según los protocolos conocidos $^{1}$, sin resultar eficaz la maniobra. Definitivamente se solucionó el cuadro en quirófano, bajo anestesia raquídea, realizando una punción con lavado de cuerpos cavernosos a través del glande. El paciente actualmente ha abandonado el tratamiento y no se ha vuelto a producir el priapismo. Los niveles de prolactina, y testosterona fueron determinados al día siguiente del priapismo, resultando los mismos normales.
La cabergolina es un derivado ergótico que actúa como agonista dopaminérgico, autorizado en España para el tratamiento de los signos y síntomas asociados a la enfermedad de Parkinson $\left(\right.$ Sogilen ${ }^{\circledR}$ ) y para tratamiento de la hiperprolactinemia e inhibición de la lactancia (Dostinex ${ }^{\circledR}$ ).

La Agencia Española de Medicamentos y Productos Sanitarios emitió en noviembre de 2004 una nota informativa sobre pergolida, otro derivado ergótico también utilizado para el tratamiento de signos y síntomas de la enfermedad de Parkinson, en relación con riesgos cariovasculares similares a los encontrados para cabergolina.

Tanto para pergolida como para cabergolina, las valvulopatías observadas se producen como consecuencia de una reacción fibrótica que produce una restricción en el movimiento normal de las válvulas, generando regurgitación que en algunos casos requiere el recambio valvular. Esta reacción fibrótica se podría producir como respuesta a la afinidad que presentan estos fármacos por los receptores serotoninérgicos 5-HT2b.

En 2 estudios publicados recientemente se ha estimado la prevalencia $^{2}$ e incidencia ${ }^{3}$ de valvulopatía cardiaca asociada al tratamiento con pergolida y cabergolina, así como de otros agonistas dopaminérgicos, comparándose con la obtenida en un grupo control. 
Zanetini et $\mathrm{al}^{2}$, en un estudio observacional en pacientes con enfermedad de Parkinson, concluyeron que la prevalencia de regurgitación valvular clínicamente relevante, en comparación con un grupo control, aumentaba de manera significativa en aquellos pacientes en tratamiento con pergolida o cabergolina, pero no en aquellos en tratamiento con otros agonistas dopaminérgicos no ergóticos. La prevalencia obtenida en este estudio para pergolida y cabergolina fue de 23,4 y $28,6 \%$ respectivamente. Estos resultados son coherentes con los obtenidos en estudios anteriores para pergolida.

En el estudio de Schade et $\mathrm{al}^{3}$, utilizando la información procedente de una base de datos de AP, se estimó un exceso de riesgo de regurgitación valvular cardiaca de nuevo diagnóstico para pergolida y cabergolina de 33 y 21 casos por cada 10.000 personas expuestas al año, respectivamente. Para los agonistas dopaminérgicos no ergóticos incluidos en el estudio no se observó incremento de riesgo en comparación con el grupo control. Asimismo, se observó que cabergolina no se asociaba con un aumento del riesgo estadísticamente significativo si se administraba a dosis de $3 \mathrm{mg}$ al día o inferiores, o durante menos de 6 meses independientemente de la dosis.

La información disponible es limitada sobre la reversibilidad de las valvulopatías tras la interrupción del tratamiento con cabergolina, así como sobre la asociación de este riesgo con el uso de cabergolina para el tratamiento de la hiperprolactinemia e inhibición de la lactancia.

Sin embargo, revisada la literatura no hemos encontrado referencia alguna sobre la relación entre el fármaco descrito y el desarrollo de cuadros de erección prolongada patológica.
La clara relación en el tiempo entre el consumo del fármaco, la aparición del priapismo, la ausencia de otros factores en este paciente que pudiesen precipitar su desarrollo, y por último, la no recurrencia de nuevos cuadros de priapismo una vez abandonado el tratamiento nos sugieren la existencia de una relación causaefecto secundario, no descrito y no deseado.

B I B L I O G R A F Í A

1. Molina L, Bejany D, Lynne CM, Politano VA. Diluted epinephrine solution for the treatment of priapism. J Urol. 1989;141:1127-8.

2. Zanettini R, Antonini A, Gatto G, et al. Valvular heart disease and the use of dopamine agonists for Parkinson's disease. N Engl J Med. 2007;356:39-46.

3. Schade R, Anderson F, Suissa S, et al. Dopamine agonists and the risk of cardiac-valve regurgitation. N Engl J Med. 2007;356:29-38 Disponible en: http://www.sen.es/pdf/2007/comunicado_valvulo patia.pdf.

\section{E. De La Peña Zarzuelo*, V. Hernández Cañas y}

C. Llorente Abarca

Servicio de Urología, Hospital Universitario Fundación Alcorcón, Madrid, España

*Autor para correspondencia.

Correo electrónico: epenaz@fhalcorcon.es

(E. De La Peña Zarzuelo). 